\title{
Research on Adaptive Track Planning Algorithm for UAV
}

\author{
Dianheng Pan ${ }^{\mathrm{a}}$, Te $\mathrm{Ma}{ }^{\mathrm{b}}$, Gang Liu ${ }^{\mathrm{c}}$ and Bing He ${ }^{\mathrm{c}}$ \\ Rocket Force University of Engineering, Xi'an 710025, China \\ apdh_0818@163.com, b382899341@qq.com, chb830513@126.com
}

Keywords: UAV, Trajectory planning, Particle Swarm, Gradient Search.

\begin{abstract}
For the low efficiency problem and the search domain widely in UAV trajectory planning, the gradient search is innovated in the traditional particle swam optimal (PSO) algorithm, then a new gradient particle swam optimization algorithm is designed by neighboring group optimization guiding population gradient. The simulation results show that the gradient particle swarm algorithm's convergence speed and accuracy is better than the traditional PSO algorithm and Genetic Algorithm.
\end{abstract}

\section{Introduction}

Unmanned Aerial Vehicle (UAV) trajectory planning is the core technology of autonomous navigation flight in a given battlefield environment. In a complex environment, UAVs take full account of the combat mission, threat deployment and maneuverability constraints of the aircraft. Utilizing terrain and enemy information, the UAV can plan a flight path from the starting point to the target point, which can be optimized or sub-optimal under certain evaluation indexes and meet various performance targets, and make the UAVs carry out threat avoidance, To enhance combat effectiveness ${ }^{[1][2]}$.

He $\mathrm{Y}^{[3]}$ used the ant colony algorithm to optimize the track of the UAV, but the test environment and the actual flight environment is very different, and seek out the track is not smooth enough, can not meet the UAV actual flight requirements . Li Sihai ${ }^{[4]}$ proposed a genetic algorithm based on the path planning method, genetic algorithm without space constraints, but may appear premature convergence and stagnation. Chen Panfeng ${ }^{[5]}$ proposed a method based on particle swarm optimization for unmanned aerial vehicle (UAV) trajectory planning. However, in the study of UAV route planning efficiency optimization, there is a slow convergence rate, the problem. Pei Xiping ${ }^{[6]}$ proposed a new algorithm of global optimization with "inertial search" as the core, but there is a drawback that the gradient descends at extremes.

In this paper, a UAV trajectory planning algorithm based on Gradient Particle Swarm Optimization (PSO) is proposed in order to plan a trajectory to meet UAV flight. This algorithm integrates the idea of gradient search into Particle Swarm Optimization (PSO), and solves the problem of premature convergence and low efficiency in traditional optimization algorithms by using the fastness of gradient search and the randomness of particle swarm search. Application in UAV route planning research.

\section{Foundation model}

When the UAV is cruising at a constant altitude during flight without taking into account the UAV's climb and descent processes, the three-dimensional flight path planning can be transformed into a two-dimensional flight path planning ${ }^{[7]}$. In this paper, UAVs are assumed to be flying at a constant altitude using this method, and the UAVs are planned based on various threats and constraints.

The track of the UAV is composed of a series of nodes in two-dimensional space, and each adjacent node can be connected by a straight line. The flight track can be expressed as $\left\{S, P_{1}, \cdots, P_{n-1}, T\right\}$, where $S$ is the starting point, $T$ is the target point, $P_{1}, \cdots, P_{n-1}$ is the middle The track node has a coordinate $\left\{x_{i}, y_{i}\right\}$.Please follow the submission instructions shown on the website.

\subsection{Track constraints.}

Due to the battlefield environment, restricted mobility and UAV combat missions, route planning need to meet certain constraints, we must consider the physical limitations of UAV and specific 
mission requirements, the paper under a two-dimensional plane, the main consideration of the minimum flight trace length constraint, the maximum turning angle constraint, the maximum track length constraints, speed constraints. Constraints on the trajectory planning, Ding Mingyue in the literature $^{[7]}$ in the above constraints the detailed description is not described here, the paper track UAV meets the constraints described by the following formula:

$$
\left\{\begin{array}{l}
g(\gamma)=0, \quad \text { Satisfy the constraints } \\
g(\gamma)=1, \quad \text { Does not satisfy the constraints }
\end{array}\right.
$$

Wherein, $\gamma$ is an ordered collection of UAV from the initial state to target state.

\subsection{Objective function design.}

Military UAV Route Planning goal is to meet the constraints and specific physical properties UAV mission constraints premise generate low altitude terrain following, terrain avoidance, threat avoidance flight path, to find the optimal route to improve the survival probability of UAV.

The establishment of track evaluation index is the basis of UAV route planning ${ }^{[8]}$, which is the key to guide the algorithm to the optimal solution approximation. The objective function using the following simplified formula:

$$
J=\sum_{i=1}^{n}\left(\omega_{1} l_{i}^{2}+\omega_{2} f_{T A i}\right)
$$

Wherein, $\omega_{1}-\omega_{2}$ is the track length and the threat index weighting, $l_{i}$ represents the length of the track section $i, f_{T A i}$ represent a threat index paragraph $i$ track segment.

The objective function is mainly on account of the total length of the track and the threat of price, on the one hand requirements shrink the total length of track section, reducing flight time and fuel savings, on the other hand to lower the threat level, and to avoid the threat of near zone.

The impact index of the UAV at point $x$ in space is affected mainly by the distance between the UAV and the threat, and the threat index $f_{T A}(x)$ of the $j$ threat to $x$ is calculated as follows ${ }^{[9]}$ :

$$
f_{T A j}(x)=\left\{\begin{array}{c}
K_{j} /\left(R_{j}\right)^{4}, x \text { is covered by threat } j \\
0, x \text { is not covered by threat } j
\end{array}\right.
$$

Wherein, $K_{j}$ is a parameter, which reflects the strength of the first $j$ threat threat sources. $R_{j}$ is the distance between the UAV and the $j$ th threat source. In the actual process, in order to reduce the amount of computation, we usually use the method mentioned in ${ }^{[9]}$ to calculate the mean value of the threat index of several points on the track segment, multiply it by length effects. Thus, if we take the first track segment dividing point $l_{i} / 5, l_{i} / 2$ and $4 l_{i} / 5$ at the threat index which can be expressed as:

$$
f_{\text {TAi }}=\frac{1}{3} \sum_{j=1}^{N_{\text {Site }}}\left\{l_{i j}\left[f_{\text {TAj }}\left(l_{i} / 5\right)+f_{\text {TAj }}\left(l_{i} / 2\right)+f_{\text {TAj }}\left(4 l_{i} / 5\right)\right]\right\}
$$

Wherein, $l_{i j}$ is the length of track $i$ in the $j$ threat coverage area, and $N_{\text {Site }}$ is the number of known threat sources.

\subsection{Mathematical Model of Route Planning.}

Based on the above described UAV planning optimization model can be summarized as follows:

$\left\{\begin{array}{lr}h(x, y)=\min _{\gamma} J(\gamma) \\ \text { s.t. } \quad g(\gamma)=0\end{array}\right.$

\section{Design of Intergranular Gradient Particle Swarm Optimization Algorithm}

\subsection{Algorithm Basic Idea.}

The traditional PSO is tracking the cost value and global best individual populations optimal solution to update the position vector and velocity vector particle by particle, this optimization algorithm PSO thought such a global search capability is weak, but also and the algorithm is easy to fall into the local optimum. In this paper, the idea of gradient search is combined with the idea of particle swarm optimization (PSO) to search the traditional particle swarm. The search gradient is 
obtained by using the global optimal value between adjacent generations. velocity vector and improve the global search ability of particle swarm optimization.

\subsection{Algorithm Basic Flow.}

The basic steps of the algorithm are as follows:

(1) Population Initialization

The first generation of random initialization vector groups and the position and velocity vector of each particle of the second-generation group, calculate fitness value of each particle, while recording the first two generations of the best individual in the population and the global optimum individual.

(2) Evaluation of Fitness

Evaluate the fitness value of each particle, if $f\left(\boldsymbol{p}_{g}^{k}\right)$ is better than $f\left(\boldsymbol{p}_{g}^{k-1}\right),(k>1)$, the step (3), otherwise step (4), where $\boldsymbol{p}_{g}{ }_{g}$ is the current generation of the best individual, $k$ is the number of iterations.

(3) Gradient Search Update

The fitness value $f\left(\boldsymbol{x}^{k}+d \boldsymbol{x}^{k}\right)$ is calculated by taking the $\alpha$ times of the global optimum position vector difference of the $k-1$ generation and the $k$ generation population as the search gradient, namely $d \boldsymbol{x}^{k}=\alpha\left(\boldsymbol{p}_{g}^{k}-\boldsymbol{p}_{g}^{k-1}\right)$. If $f\left(\boldsymbol{x}^{k}+d \boldsymbol{x}^{k}\right)$ is better than $f\left(\boldsymbol{x}^{k}\right)$, the search succeeds and the search is continued along the gradient direction; otherwise the search is finished. Which can get the second group of the first $k$ generation global best individual optimization solution $\left(\boldsymbol{p}_{g}^{k}\right)^{\prime}$, so $\boldsymbol{p}_{g}^{k}=\left(\boldsymbol{p}_{g}^{k}\right)^{\prime}$.

Wherein $\alpha$ is a positive constant, $\alpha$ bigger, stronger global search ability of the algorithm, $\alpha$ smaller, local search algorithm is the stronger. $\alpha$ 's value strategy is:

$$
\alpha(k)=\alpha_{\max }-\frac{\alpha_{\max }-\alpha_{\min }}{k_{\max }} \times k
$$

(4) Generation of New Generation Population

The algorithm takes the current optimal position $\boldsymbol{p}_{g}^{k}$ of population $k$ as a starting point to generate a new generation population. The updating formula of position vector and velocity vector is shown as formula (7). In the formula, inertia weight adopts linear descending weight and learning factor adopts asynchronous change learning factor [10].

$$
\left\{\begin{array}{c}
v_{i j}^{k+1}=\omega v_{i j}^{k}+c_{1} r_{1}\left(p_{i j}^{k}-x_{i j}^{k}\right)+c_{2} r_{2}\left(p_{g j}^{k}-x_{i j}^{k}\right) \\
x_{i j}^{k+1}=x_{g j}^{k}+v_{i j}^{k+1}
\end{array}\right.
$$

(5) The Termination Condition Determination

It is judged whether the optimization result reaches the given precision or whether the iteration number reaches the given value. If you meet the given conditions, the iteration is terminated, and output the optimal solution, otherwise, go to (2).

The optimization process of the algorithm shown in Fig. 1:

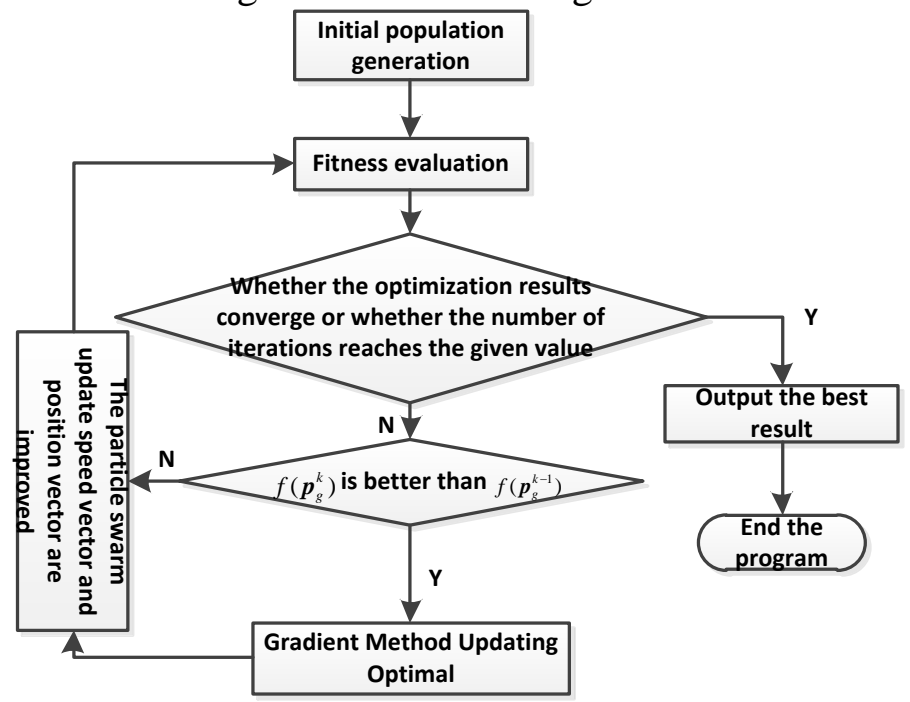

Fig. 1 Flow chart of UAV route planning optimization 


\section{Simulation and Analysis of Results}

\subsection{Algorithm Basic Idea.}

Track starting point coordinate $(5,5)$, the end point coordinates $(1023,1023)$, the threat source is set to 8 , the threat represented by A circular area, B denotes the center position coordinates of the sources of threats, $\mathrm{C}$ threat sources effective radius, the threat distributed as follows:

Table 1. Threat Distribution

\begin{tabular}{ccc}
\hline Threat Sources & Center Coordinates $/(\mathrm{km}, \mathrm{km})$ & Effective Radius/ $\mathrm{km}$ \\
\hline Radar 1 & $(155,265)$ & 75 \\
Artillery 1 & $(310,565)$ & 60 \\
Radar 2 & $(371,161)$ & 107 \\
Artillery 2 & $(459,770)$ & 105 \\
Artillery 3 & $(514,530)$ & 40 \\
Radar 3 & $(657,664)$ & 66 \\
Artillery 4 & $(790,449)$ & 100 \\
Radar 4 & $(903,920)$ & 62 \\
\hline
\end{tabular}

Based on the hybrid PSO algorithm proposed in this paper, the UAV route planning is optimized. Particle size group taking 200, 250 take the maximum number of iterations, the inertia weight maximum is 0.9 , the minimum is 0.4 , the initial value of the learning factor 2.5 , the termination value of 0.5 , the initial learning factor of 0.5 , the termination value of 2.5 , the initial value of $2.5,0.5$ is terminated.

\subsection{Algorithm Comparison Analysis.}

Based on the given parameters, the simulation 50 times, route planning results shown in Fig. 2, based on intergenerational UAV track gradient particle swarm optimal planning of individual and population average cost value curve shown in Fig. 3 as follows:

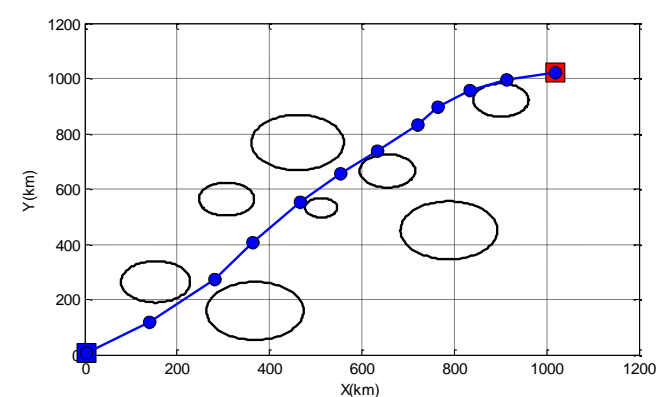

Fig. 2 UAV Trajectory Planning Based on Improved Objective Function

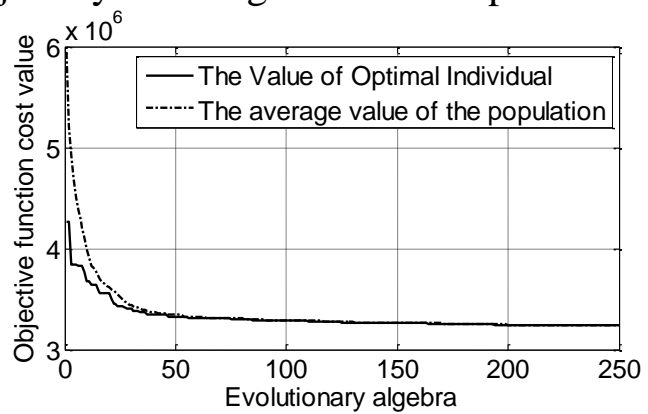

Fig. 3 Curve of the Change of Route Planning Cost Based on Intergenerational Gradient Particle Swarm

The convergence curves of the genetic algorithm, particle swarm algorithm and gradient particle swarm optimization algorithm are shown in Fig.4: 


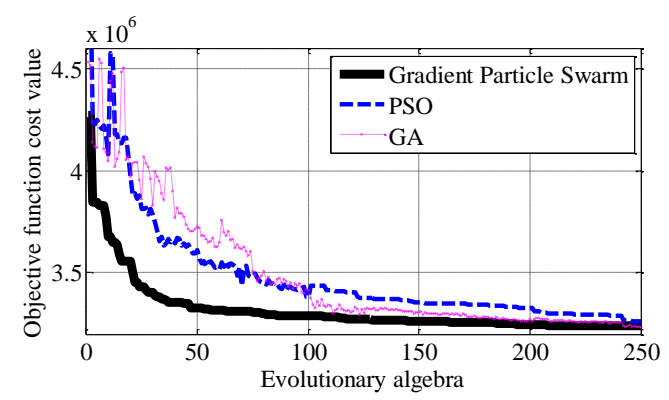

Fig. 4 Comparison of evolutionary processes of algorithms

Through the optimal algorithm convergence curve comparison, gradient particle swarm algorithm convergence rate significantly higher than the other two algorithms, but accuracy has not been greatly improved. In order to compare the performance of each algorithm, 50 times the average of the simulation results of the statistics, the results are as follows:

Table 2. Compares the Performance of the Algorithm

\begin{tabular}{ccc}
\hline Algorithm Name & Convergence Accuracy & Convergence Rate \\
\hline Gradient Particle Swarm & $3.2322 \times 106$ & 78.314979 \\
PSO & $3.9647 \times 106$ & 138.401417 \\
GA & $3.6383 \times 106$ & 176.791815 \\
\hline
\end{tabular}

When obtaining UAV route planning, this paper introduces the gradient than the traditional particle swarm optimization algorithm convergence of high precision, gradient particle swarm genetic algorithm relative increase $11.16 \%$, the relative particle swarm increase $18.47 \%$; particle swarm algorithm convergence velocity gradient fastest gradient particle swarm genetic algorithm relative increase $55.70 \%$, the relative particle swarm increase 43.51 percent.

The Gradient Particle Swarm Optimization (PSO) algorithm introduced in this paper combines the characteristics of the gradient search to make the particle population jump out of the local optimal solution, which solves the problem that the PSO is easy to "premature". The simulation results show that the algorithm of global optimization and convergence rate than the traditional genetic algorithm and particle swarm optimization has improved significantly.

\section{Summary}

In this paper, the background of UAV route planning, route planning model established tradition, since a wider search area of the model, resulting in reduced efficiency of traditional search optimization, this paper designed an inter-generation gradient particle swarm optimization. The simulation results show that the algorithm compared to traditional optimization algorithm has higher convergence speed and precision for UAV route planning.

\section{References}

[1]. Zhang Lei, Fang Yangwang, Chai Dong, et al. Improved cruise missile route planning method based on improved quantum evolutionary algorithm [J]. Journal of Ordnance Engineering. Vol. 35 (2014) No. 11, p. 1820-1827.

[2]. Shen Lincheng, Chen Jing, Wang Nan. Review of aircraft mission planning [J]. Journal of Aeronautical, 2014, 35(3): 593-606.

[3]. He Y, Zeng Q, Liu J, et al. Path planning for indoor UAV based on Ant Colony Optimization[C]//2013 25th Chinese Control and Decision Conference (CCDC). IEEE, 2013: 2919-2923.

[4]. Li Sihai, Bai Cunru. Research on Route Planning of Aircraft Based on Genetic Algorithm [J]. Journal of East China Jiaotong University, 2007, 24 (4): 147-151. 
[5]. CHEN Pan-feng, LIU Wen-jun, WANG Wei. Path Planning of UAV Based on Particle Swarm Optimization Algorithm [J]. Electronic Design \& Engineering, 2013, 21 (22): 36-39.

[6]. Pei Xiping, Hao Xiaogong, Chen Wei, et al. Optimization Design of Single Phase Amplitude Phase Locked Loop Based on Gradient Descent Method [J]. Automation of Electric Power Systems, 2014, 2: 019.

[7]. Ding Mingyue. Unmanned Aerial Vehicle Track Planning [M]. Electronic Industry Press, 2009: 39-44.

[8]. Xie Xiaofang, Sun Tao, Ouyang Zhonghui. Anti-ship missile route planning technology [M]. Beijing: National Defense Industry Press, 2010: 23-30.

[9]. Beard R W, McLain T W, Goodrich M A, et al. Coordinated target assignment and intercept for unmanned air vehicles[J]. IEEE transactions on robotics and automation, 2002, 18(6): 911-922.

[10]. Yu Lei. MATLAB intelligent algorithm 30 case analysis [M]. Beijing University of Aeronautics and Astronautics Press, 2015:102-107. 